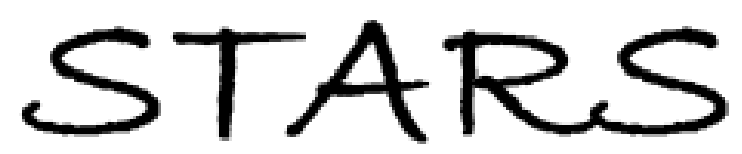

University of Central Florida

STARS

Faculty Bibliography 2010s

Faculty Bibliography

$1-1-2010$

\title{
PT-symmetric optical lattices
}

Konstantinos G. Makris

University of Central Florida

Ramy El-Ganainy

University of Central Florida

Demetrios N. Christodoulides

University of Central Florida

Z. H. Musslimani

Find similar works at: https://stars.library.ucf.edu/facultybib2010

University of Central Florida Libraries http://library.ucf.edu

This Article is brought to you for free and open access by the Faculty Bibliography at STARS. It has been accepted for inclusion in Faculty Bibliography 2010 s by an authorized administrator of STARS. For more information, please contactSTARS@ucf.edu.

\section{Recommended Citation}

Makris, Konstantinos G.; El-Ganainy, Ramy; Christodoulides, Demetrios N.; and Musslimani, Z. H., "PTsymmetric optical lattices" (2010). Faculty Bibliography 2010s. 493.

https://stars.library.ucf.edu/facultybib2010/493

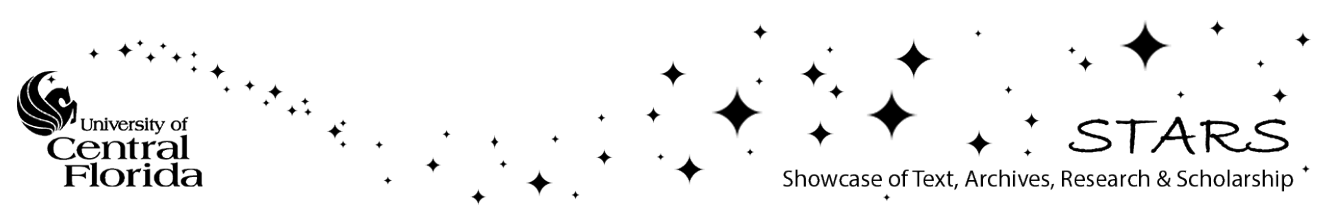




\title{
$\mathcal{P} \mathcal{T}$-symmetric optical lattices
}

\author{
Konstantinos G. Makris, Ramy El-Ganainy, and Demetrios N. Christodoulides \\ College of Optics/CREOL, University of Central Florida, Orlando, Florida 32816, USA \\ Z. H. Musslimani \\ Department of Mathematics, Florida State University Tallahassee, Florida 32306-4510, USA
}

(Received 15 November 2009; published 4 June 2010)

\begin{abstract}
The basic properties of Floquet-Bloch $(\mathrm{FB})$ modes in parity-time $(\mathcal{P} \mathcal{T})$-symmetric optical lattices are examined in detail. Due to the parity-time symmetry of such complex periodic potentials, the corresponding FB modes are skewed (nonorthogonal) and nonreciprocal. The conjugate pairs of these FB modes are obtained by reflecting both the spatial coordinate and the Bloch momentum number itself. The orthogonality conditions are analytically derived for a single cell, for both a finite and an infinite lattice. Some of the peculiarities associated with the diffraction dynamics in $\mathcal{P} \mathcal{T}$ lattices such as nonreciprocity, power oscillations, and phase dislocations, are also examined.
\end{abstract}

DOI: 10.1103/PhysRevA.81.063807

PACS number(s): 42.25.Bs, 11.30.Er, 42.82.Et

\section{INTRODUCTION}

The correspondence between operators and physical quantities is crucial within the context of quantum mechanics. Since any physically measurable quantity must always be real, the eigenvalues of every quantum operator corresponding to it should also belong to the real set. This leads to one of the basic postulates of quantum theory, namely that every observable can only be associated with a Hermitian (selfadjoint) operator. It was not until recently, that the condition for Hermiticity, as an absolute prerequisite for real spectrum, was critically reexamined [1]. In a pioneering study, Bender et al. suggested that even non-Hermitian Hamiltonians can still have an entirely real eigenvalue spectrum provided that they respect parity-time $(\mathcal{P} \mathcal{T})$ symmetry. In addition, they demonstrated that such Hamiltonians can also undergo a sudden phase transition (spontaneous $\mathcal{P} \mathcal{T}$ symmetry-breaking) above a critical threshold. In other words, above this transition point the eigenvalue spectrum ceases being entirely real and becomes instead partially complex. The extension of these concepts into the complex domain has been the topic of several studies during the last few years [2-5].

A Hamiltonian is called $\mathcal{P} \mathcal{T}$ symmetric, if and only if, it has the same eigenfunctions with that of the $\mathcal{P} \mathcal{T}$ operator [2]. Here the action of the parity operator $\hat{P}$ is defined by the relations $\hat{p} \rightarrow-\hat{p}, \quad \hat{x} \rightarrow-\hat{x}$, whereas that of the time operator $\hat{T}$ by $\hat{p} \rightarrow-\hat{p}, \quad \hat{x} \rightarrow \hat{x}, \quad i \rightarrow-i$, where $\hat{p}, \hat{x}$ are the momentum and position operators, respectively. In operator form, the normalized Schrödinger evolution equation $(\hbar=m=1)$ is given by $i \Psi_{t}=\hat{H} \Psi$, where $\hat{H}=\hat{p}^{2} / 2+V(\hat{x})$ and $\hat{p} \rightarrow$ $-i \partial / \partial x$ [6]. Given that the $\hat{T}$ operation corresponds to a time reversal, i.e., $\hat{T} \hat{H}=\hat{p}^{2} / 2+V^{*}(x)$, one can then deduce that $\hat{H} \hat{P} \hat{T}=\hat{p}^{2} / 2+V(x)$ and $\hat{P} \hat{T} \hat{H}=\hat{p}^{2} / 2+V^{*}(-x)$. Based on the previous considerations, it can be directly shown that a necessary (but not sufficient) condition for a Hamiltonian to be $\mathcal{P} \mathcal{T}$ symmetric is $V(x)=V^{*}(-x)$. In other words, the real part of the complex potential involved must be a symmetric function of position, while the imaginary component should be antisymmetric.

The fact that wave quantum mechanics and scalar paraxial optics are described by the same mathematical equation, often leads to formal analogies between these two distinct areas of physical science. In this context, complex optical potentials can be realized by using gain and loss spatial distributions. In particular, there have been several studies dealing with optical wave propagation in complex potentials at both the theoretical and experimental level. Examples include, gain guiding in waveguides [7], Ginzburg-Landau solitons [8], and wave scattering from complex periodic potentials $[9,10]$.

Quite recently the notion of $\mathcal{P} \mathcal{T}$ symmetry was introduced within the framework of optics [11-16]. This suggestion was based on judicious designs that involve both optical gain/loss regions and the process of index guiding. Optical potentials satisfying the necessary condition for $\mathcal{P} \mathcal{T}$ symmetry were considered in order to synthesize a new class of materials with properties and characteristics that have no analog in standard gain/guiding structures. Along these lines, periodic arrangements or waveguide arrays $[17,18]$ composed of $\mathcal{P} \mathcal{T}$ symmetric cells can be envisioned [12,13], where the entire structure respects parity-time symmetry. Since the eigenmodes of such systems are not orthogonal, their coupled mode theories must be reformulated starting from variational principles as shown in [11]. Moreover, the beam dynamics, as well as, the stationary properties of such $\mathcal{P} \mathcal{T}$ symmetric optical lattices were theoretically studied in both 1D and 2D spatial dimensions [12]. Nonreciprocal behavior, band merging, double refraction and abrupt phase transitions, are some of the exotic features associated with these periodic potentials. In the nonlinear domain, one and two-dimensional soliton solutions were found to exist below and above the phase transition point, in nonlinear $\mathcal{P} \mathcal{T}$ lattices [13]. The interplay of the Kerr nonlinearity with the $\mathcal{P} \mathcal{T}$ threshold was analyzed for the first time [13], and analytical periodic solutions were also derived [14]. Most importantly, the first experimental observation of the $\mathcal{P} \mathcal{T}$ symmetry in any physical system has been recently achieved in the framework of wave optics [15,16]. In particular, both "passive" [15] and "active" [16] $\mathcal{P} \mathcal{T}$ optical couplers have experimentally demonstrate inherent characteristics of these pseudo-Hermitian systems, such as abrupt phase transitions, and nonorthogonal supermodes. 
In this paper, we investigate in detail the band structure and wave dynamics related to complex $\mathcal{P} \mathcal{T}$ symmetric arrays. In particular, the nonreciprocal and nonorthogonal nature of the corresponding Floquet-Bloch (FB) modes is analytically and numerically demonstrated. Since this class of FB modes is nonorthogonal in the usual sense, a new inner product is derived based on variational arguments. The conjugate pairs of these FB modes are obtained by reflecting both the spatial coordinate and the Bloch momentum vector itself. The orthogonality conditions are analytically proved for a single cell, a finite and an infinite lattice, along with the projection coefficients and the completeness relations. Some of the peculiarities of the diffraction dynamics arising in $\mathcal{P} \mathcal{T}$ lattices such as nonreciprocity, power oscillations, and phase dislocations are also discussed.

\section{BAND STRUCTURE AND FB MODES OF A $\mathcal{P} \mathcal{T}$-OPTICAL LATTICE}

The analogy between quantum mechanics and optics is based on the fact that they share the same mathematical formalism. More specifically, the equation governing optical beam propagation is described by a Schrödinger-like equation, namely the paraxial equation of diffraction. Here we will primarily explore the diffraction dynamics of optical beams and waves in $\mathcal{P} \mathcal{T}$-symmetric complex potentials in the spatial domain. In normalized units, the propagation dynamics is described by

$$
i \frac{\partial \psi}{\partial z}+\frac{\partial^{2} \psi}{\partial x^{2}}+V(x) \psi=0,
$$

where $\psi$ represents the electric field amplitude, $z$ the propagation distance, $x$ the spatial coordinate, and $V(x)$ the complex periodic optical potential with period $D, V(x)=V(x+D)$. From the previous discussion, this complex potential is $\mathcal{P} \mathcal{T}$ symmetric provided that its real part or refractive index profile is even, while the imaginary component (loss and gain profile) is odd, e.g., $V(x)=V^{*}(-x)$.

Let us first consider the characteristics of the band structure corresponding to a periodic $\mathcal{P} \mathcal{T}$ structure. To do so, we look for stationary solutions of the form $\phi_{k n}(x) \exp \left[i \beta_{n}(k) z\right]$, where $\phi_{k n}(x)$ is the $n$-band Floquet-Bloch mode with Bloch momentum $k$, and $\beta_{n}(k)$ is the corresponding eigenvalue or propagation constant. In all cases the Bloch wave vector $k$ is restricted in the first Brillouin zone, $-\pi / D \leqslant k<\pi / D$. Without any loss of generality, let us consider the periodic $\mathcal{P} \mathcal{T}$ potential $V(x)=4\left[\cos ^{2}(x)+i V_{0} \sin (2 x)\right]$, with period $D=$ $\pi$. The real (solid line) and the imaginary part (dotted line) of the $V(x)$ potential for $V_{0}=0.3$ are depicted in Fig. 1(a). We emphasize once more, that the condition $V(x)=V^{*}(-x)$ satisfied by this potential is a necessary but not a sufficient condition for the reality of the eigenvalue spectrum. By using spectral techniques we numerically determine the existence of a $\mathcal{P} \mathcal{T}$ threshold $\left(V_{0}^{\text {th }}\right)$, below which all the propagation
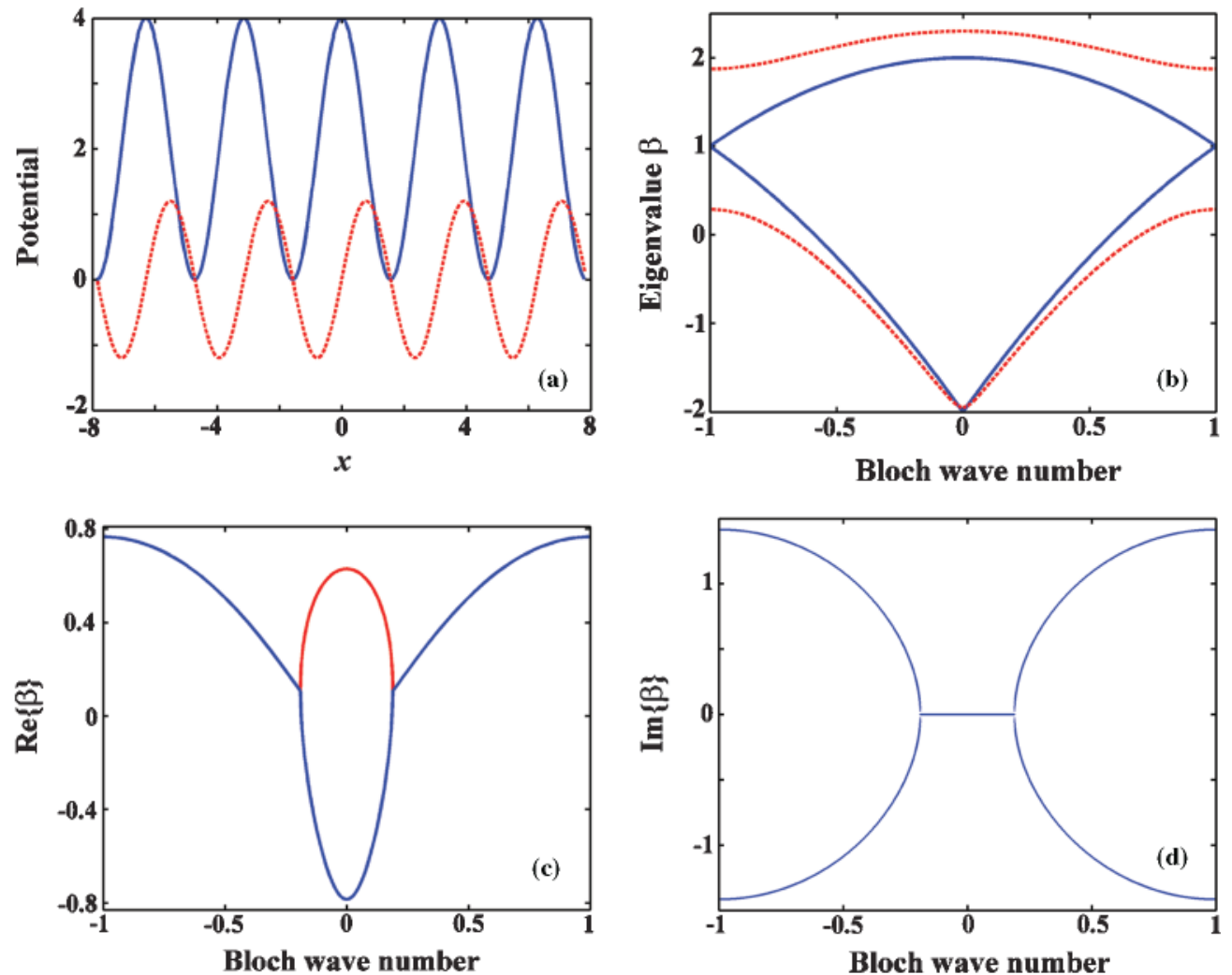

FIG. 1. (Color online) (a) Real (solid line) and imaginary (dotted line) component of a $\mathcal{P} \mathcal{T}$-symmetric lattice with $V_{0}=0.3$. (b) The real part of the first two bands of the same potential for different values of gain/loss amplitude $V_{0}=0.3$ (solid-dotted lines) and $V_{0}=$ 0.5 (solid lines). (c) Real part of the first two bands for $V_{0}=0.85$, and (d) the corresponding imaginary parts of the bands presented in (c). 
eigenvalues for every band and every Bloch wave number $k$ are real. Above this $\mathcal{P} \mathcal{T}$ threshold, an abrupt phase transition [19] occurs and as a result the spectrum is partially complex in spite of the fact that $V(x)=V^{*}(-x)$ is still valid. For the specific potential assumed here we find a threshold of $V_{0}^{\text {th }}=0.5$. In other words, for $V_{0}<0.5$, the band structure is entirely real while for $V_{0}>0.5$ it becomes partially complex. Figure 1(b) illustrates the first two bands of this potential for two cases, i.e., when $V_{0}=0.3$ (solid-dotted lone) and $V_{0}=0.5$ (solid line). Note that below $V_{0}^{\text {th }}$ all the forbidden gaps are open whereas at the threshold $V_{0}^{\text {th }}=0.5$ the first band gap at the edges of the Brillouin zone $k= \pm 1$ closes, as shown in Fig. 1(b). Moreover, when $V_{0}$ exceeds this critical value the first two bands start to merge together forming an oval-like double-valued band with an associated complex spectrum. The real as well as the imaginary parts of such a band are depicted in Figs. 1(c) and 1(d), respectively, when $V_{0}=0.85$. These
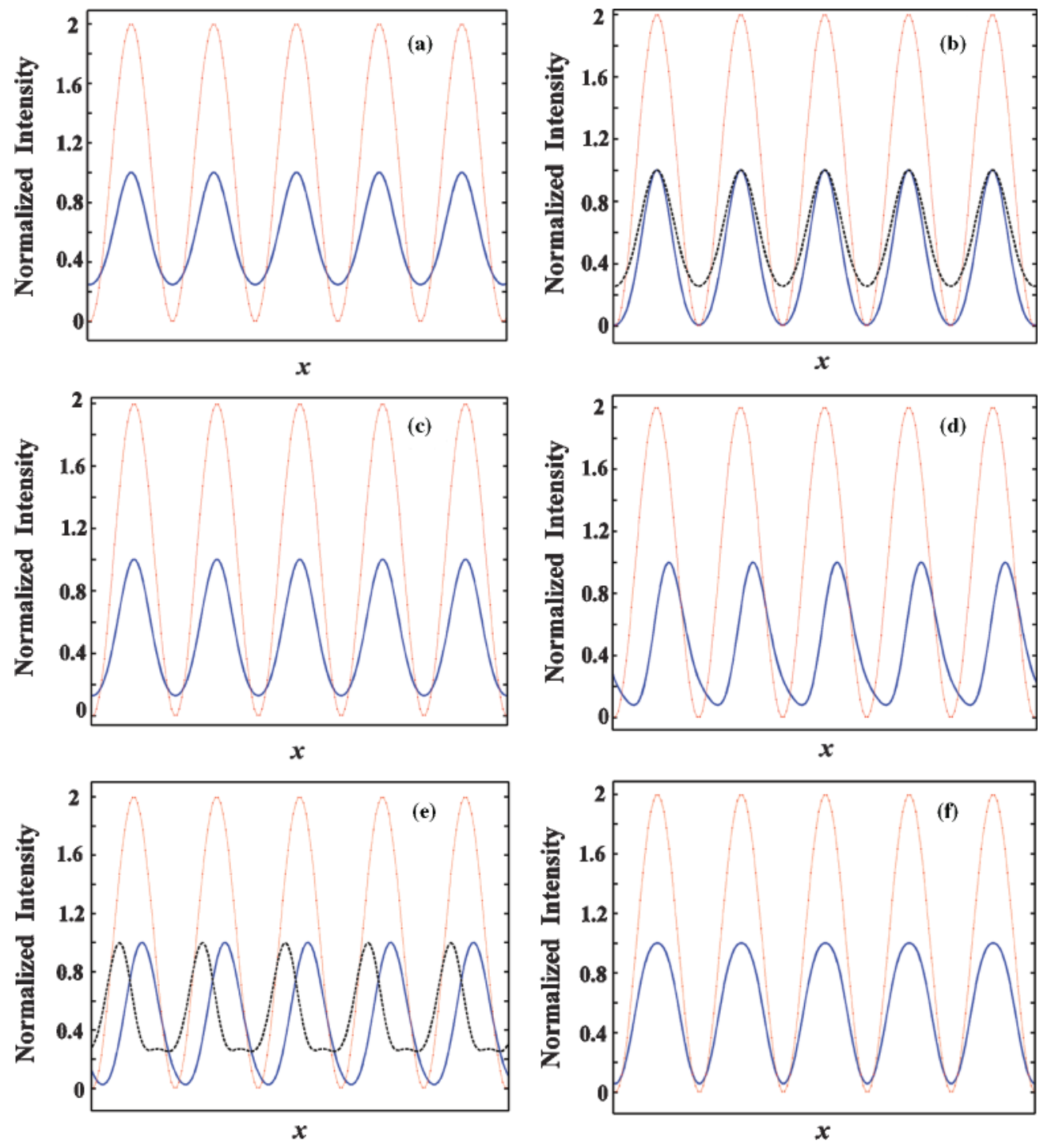

FIG. 2. (Color online) Normalized intensity profiles of FB modes of the first band of a $\mathcal{P} \mathcal{T}$ symmetric lattice for different values of $V_{0}$, and Bloch wave numbers $k$. Below the $\mathcal{P} \mathcal{T}$ threshold for $V_{0}=0.49$, FB modes with the following Bloch momentum are presented (a) $k=-1$, (b) $k=0.5$ (dashed line), $k=-0.5$ (solid line), (c) $k=0$. Above the phase transition point for $V_{0}=0.85$, we have (d) $k=-1$, (e) $k=0.5$ (FB mode of the second band) (dashed line), $k=-0.5$ (solid line), (f) $k=0$. In all cases, the real part of the periodic potential (solid-dotted) line is schematically depicted. 
eigenfunctions have no zero nodes at $k= \pm \pi / D= \pm 1$ (edge of the Brillouin zone) [4], as we can clearly see in Fig. 2(a). Above the phase transition point, the FB modes with the same eigenvalue $\beta_{n}(k)$ form complex conjugate pairs, the same way the first two bands do. One of these modes at $k=-1$ of the first band is shown in Fig. 2(d), where the spatial shift toward the loss region is apparent. The complex conjugate pair of this FB mode belongs to the second transmission band and is spatially shifted toward the gain region. An example of such a FB mode of the second band is depicted in Fig. 2(e) for the case of $k=0.5$. We point out that, unlike in real lattices, a FB mode with Bloch wave number $k$ is not equal to the complex conjugate of the FB mode at $-k$, i.e., $\phi_{k n}(x) \neq \phi_{-k n}^{*}(x)$. For this reason we say that a band structure in a parity-time symmetric potential is nonreciprocal, with respect to the Bloch momentum $k$. Bloch modes corresponding to $\pm k$ wave numbers have completely different field profiles, as Figs. 2(b) and 2(e) demonstrate.

We emphasize that these peculiar features are a direct consequence of the nonorthogonality of the $\mathcal{P} \mathcal{T}$ FloquetBloch functions. In fact, the usual orthogonality condition $\int_{-\infty}^{+\infty} \phi_{k^{\prime} m}^{*}(x) \phi_{k n}(x) d x=\delta_{n m} \delta\left(k-k^{\prime}\right)$ (that is valid in real crystals) is no longer observed in $\mathcal{P} \mathcal{T}$ symmetric lattices. This skewness of the FB modes [12] is an inherent characteristic of $\mathcal{P} \mathcal{T}$ symmetric periodic potentials and has important consequences on their algebra. Thus a more convenient reciprocal basis has to be developed for the inner product. This new inner product should be capable of projecting any input wave function on the skewed FB eigenmode basis.

\section{INNER PRODUCT ALGEBRA FOR $\mathcal{P} \mathcal{T}$-PERIODIC POTENTIALS}

In standard Hermitian optics (when only real potentials are involved), the inner product of two complex-valued functions is generally defined as $\langle h, g\rangle=\int_{-\infty}^{+\infty} h^{*}(x) g(x) d x$. Accordingly, the orthogonality relation between two different FB modes, $\phi_{k n}(x), \phi_{k^{\prime} m}(x)$ corresponding to a periodic potential, is given by $\int_{-\infty}^{+\infty} \phi_{k^{\prime} m}^{*}(x) \phi_{k n}(x) d x=\delta_{n m} \delta\left(k-k^{\prime}\right)$. The question naturally arises as to how one could define an inner product for a complex $\mathcal{P} \mathcal{T}$-symmetric lattice. In order to answer this question we first have to obtain the conjugate pairs associated with the corresponding Lagrangian. This can be done by substituting a FB mode profile $\phi_{k n}(x) \exp \left[i \beta_{n}(k) z\right]$ in Eq. (1). By doing so we get the following linear eigenvalue problem:

$$
\phi_{k n}^{\prime \prime}(x)+V(x) \phi_{k n}(x)=\beta_{n}(k) \phi_{k n}(x) .
$$

The Lagrangian associated with the above equation is given by

$$
\begin{aligned}
L_{1}= & {\left[\partial_{x} \phi_{k n}(x)\right]\left[\partial_{x} \phi_{k n}^{*}(-x)\right]-\left[V(x)-\beta_{n}(k)\right] } \\
& \times \phi_{k n}(x) \phi_{k n}^{*}(-x),
\end{aligned}
$$

where $\partial_{x} \phi(x) \equiv \frac{\partial \phi(x)}{\partial x}$. It is straightforward to show that Euler-Lagrange variations on (3) $\frac{\partial}{\partial x}\left(\frac{\partial L_{1}}{\partial\left[\partial_{x} \phi_{k n}(x)\right]}\right)-\frac{\partial L_{1}}{\partial \phi_{k n}(x)}=$ $0, \frac{\partial}{\partial x}\left(\frac{\partial L_{1}}{\partial\left[\partial_{x} \phi_{k n}^{*}(-x)\right]}\right)-\frac{\partial L_{1}}{\partial \phi_{k n}^{*}(-x)}=0$ indeed lead to Eq. (2) under the condition of $\mathcal{P} \mathcal{T}$ symmetry $V(x)=V^{*}(-x)$. As a result the conjugate pairs of the Eq. (2) are $\phi_{k n}(x), \phi_{k n}^{*}(-x)$ and the corresponding new inner product should be defined as $\langle h, g\rangle=$ $\int_{-\infty}^{+\infty} h^{*}(-x) g(x) d x$. Since the Floquet-Bloch theorem is valid for all periodic potentials (real and complex), it follows that every FB mode can be written as $\phi_{k n}(x)=u_{k n}(x) \exp (i k x)$ where $u_{k n}(x)=u_{k n}(x+D)$, and $D$ is the normalized period of the complex $\mathcal{P} \mathcal{T}$-symmetric periodic potential $V(x)$. Consequently, direct substitution shows that Eq. (2) is equivalent to

$$
u_{k n}^{\prime \prime}+2 i k u_{k n}^{\prime}+\left[V(x)-k^{2}\right] u_{k n}=\beta_{n}(k) u_{k n}
$$

with the following Lagrangian:

$$
\begin{aligned}
L_{2}= & {\left[\partial_{x} u_{k n}(x)\right]\left[\partial_{x} u_{-k n}^{*}(-x)\right]-\left[V(x)-k^{2}-\beta_{n}(k)\right] u_{k n}(x) } \\
& \times u_{-k n}^{*}(-x)+2 i k u_{k n}(x)\left[\partial_{x} u_{-k n}^{*}(-x)\right]
\end{aligned}
$$

Apparently the conjugate pairs of Eq. (5) are now different. In particular they are $u_{k n}(x), u_{-k n}^{*}(-x)$ and the corresponding inner product is $\left\langle h_{k}, g_{k}\right\rangle=\int_{-\infty}^{+\infty} h_{-k}^{*}(-x) g_{k}(x) d x$. It is important to note that, for the conjugate variable, not only do we have to invert the spatial coordinate $x$, but the Bloch wave number $k$ must be inverted as well. Since this inner product is different than that employed in a real lattice, the orthogonality condition is also expected to take a different form and must be systematically derived. As mentioned before, this is accomplished by first considering the orthogonality in a single cell, a finite lattice, and at the end examine the case of an infinite lattice. In all these three cases we refer always to the eigenvalue problem of Eq. (4).

\section{ORTHOGONALITY IN A SINGLE $\mathcal{P} \mathcal{T}$-CELL}

The goal of this paragraph is to derive the orthogonality condition in one individual cell of the periodic potential. This cell is defined in the range $-D / 2 \leqslant x \leqslant D / 2$. Let us consider two FB modes $u_{k n}, u_{k m}$ belonging to different bands $(n \neq m)$ but having the same wave number $k$. We also assume that the potential $V(x)$ is used below the $\mathcal{P} \mathcal{T}$-phase transition point, and therefore the eigenvalue spectrum is entirely real. Then from Eq. (4) we get the following equations for these two modes:

$$
\begin{gathered}
u_{k n}^{\prime \prime}+2 i k u_{k n}^{\prime}+\left[V(x)-k^{2}\right] u_{k n}=\beta_{n}(k) u_{k n}, \\
u_{-k m}^{\prime \prime *}-2 i k u_{-k m}^{\prime *}+\left[V^{*}(-x)-k^{2}\right] u_{-k m}^{*}=\beta_{m}(-k) u_{-k m}^{*},
\end{gathered}
$$

where $u_{-k m}^{*} \equiv u_{-k m}^{*}(-x)$. By multiplying the first one with $u_{-k m}^{*}$ and the second one with $u_{k n}$ and by taking into account that $V(x)=V^{*}(-x)$, we have

$$
\begin{aligned}
& u_{-k m}^{*} u_{k n}^{\prime \prime}+2 i k u_{-k m}^{*} u_{k n}^{\prime}+\left[V(x)-k^{2}\right] u_{-k m}^{*} u_{k n} \\
& \quad=\beta_{n}(k) u_{-k m}^{*} u_{k n} \\
& u_{k n} u_{-k m}^{\prime \prime *}-2 i k u_{k n} u_{-k m}^{\prime *}+\left[V^{*}(-x)-k^{2}\right] u_{k n} u_{-k m}^{*} \\
& \quad=\beta_{m}(-k) u_{k n} u_{-k m}^{*} .
\end{aligned}
$$

By subtracting and taking the integral over the whole cell, we obtain $\left[\beta_{n}(k)-\beta_{m}(-k)\right] \int_{-D / 2}^{D / 2} u_{-k m}^{*} u_{k n} d x=0$, where we have used the periodicity of $u_{k n}$, e.g., $u_{k n}(-D / 2)=u_{k n}(D / 2)$. If we allow $n=m$, we get $\left[\beta_{n}(k)-\beta_{n}(-k)\right] \int_{-D / 2}^{D / 2} u_{-k n}^{*}(-x) u_{k n}(x) d x=0$ and since $\int_{-D / 2}^{D / 2} u_{-k n}^{*}(-x) u_{k n}(x) d x \neq 0$ for all FB modes (checked numerically), we obtain $\beta_{n}(k)=\beta_{n}(-k)$. This last relation 
implies that in a $\mathcal{P} \mathcal{T}$-symmetric lattice all the corresponding bands are symmetric around the center of the Brillouin zone $k=0$. Furthermore, since there is no degeneracy $\beta_{m}(k) \neq \beta_{n}(k)$, and by using the symmetry of the band structure, we finally get the orthogonality condition over a single cell in a periodic potential, that is

$$
\int_{\text {cell }} u_{-k m}^{*}(-x) u_{k n}(x) d x=0 .
$$

Next we define the normalized FB modes as $\Phi_{k n}(x) \equiv$ $\phi_{k n}(x) / \sqrt{c_{k n}}$, and $U_{k n}(x) \equiv u_{k n}(x) / \sqrt{c_{k n}}$ where $c_{k n} \equiv$ $\int_{-D / 2}^{D / 2} \phi_{-k n}^{*}(-x) \phi_{k n}(x) d x$. It is easy to see that every FB function has a unique normalization coefficient $c_{k n}$ depending on the band index $n$ and the Bloch wave number $k$. In general $c_{k n}$ are complex numbers and satisfy the symmetry relation $c_{k n}=c_{-k n}^{*}$. Based on this normalization, Eq. (6) leads to the final orthonormality condition, which is

$$
\int_{\text {cell }} \Phi_{-k m}^{*}(-x) \Phi_{k n}(x) d x=d_{k n} \delta_{n, m},
$$

where $d_{k n} \equiv\left\{\begin{array}{c}1, \text { when } c_{k n} \in C \text { or } c_{k n}>0 \\ -1, \text { when } c_{k n}<0\end{array}\right.$ and $\delta_{n, m}$ is the Kronecker delta. As stated before, it has been numerically verified that for all FB modes below the $\mathcal{P} \mathcal{T}$ phase-transition point $c_{k n} \neq 0$. In other words no self-orthogonal FB modes exist in our problem [20] and the above normalization is indeed well defined.

\section{ORTHOGONALITY IN INFINITE $\mathcal{P} \mathcal{T}$ LATTICES}

Here we derive the orthonormality condition of two FB modes in a $\mathcal{P} \mathcal{T}$-symmetric infinite optical lattice. In order to do this, we calculate the corresponding inner product in a finite lattice of $N$ cells and then take the limit $N \rightarrow \infty$. For this purpose we consider a finite lattice with an odd number of waveguides $N=2 N^{\prime}+1$ where $N^{\prime}$ is the number of cells to the left and to the right of the central element. The width of the array is given by $L=N D$ where $D$ is the lattice constant. First, we reduce the calculation of the inner product to an integral over just one (the central) cell, as opposed to over the entire lattice. In particular, we split the integral in every individual cell in the following fashion:

$$
\begin{aligned}
& \int_{\substack{\text { finite } \\
\text { lattice }}} \Phi_{-k^{\prime} m}^{*}(-x) \Phi_{k n}(x) d x \\
& =\int_{\substack{\text { finite } \\
\text { lattice }}} U_{-k^{\prime} m}^{*}(-x) U_{k n}(x) \exp \left[i\left(k-k^{\prime}\right) x\right] d x
\end{aligned}
$$

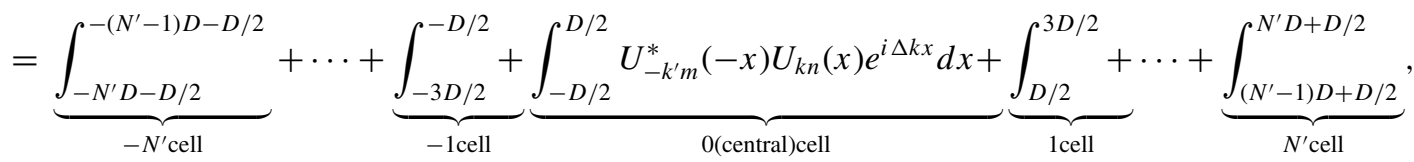

where we used $\Delta k \equiv k-k^{\prime}$ and $\Phi_{k n}(x)=U_{k n}(x) \exp (i k x)$. Given the fact that $u_{k n}(x)$ is periodic with period $D$, every term in the summation can be reduced to an integral over the central cell by using the change of variables $x=x^{\prime}-p D$ with $p= \pm 1, \pm 2, \ldots, \pm N^{\prime}$ :

$$
\begin{aligned}
& \int_{\substack{\text { finite } \\
\text { lattice }}} \Phi_{-k^{\prime} m}^{*}(-x) \Phi_{k n}(x) d x \\
& =\int_{-D / 2}^{D / 2} U_{-k^{\prime} m}^{*}(-x) U_{k n}(x) e^{i \Delta k x} d x \cdot\left[\sum_{j=-N^{\prime}}^{N^{\prime}} e^{i \Delta k D j}\right] .
\end{aligned}
$$

Notice that the sum of the above geometric series is wellknown in Fourier analysis [21] as the Dirichlet kernel $D_{N}(x) \equiv$ $\sum_{m=-N}^{N} e^{i x m}, x \in R$, and thus the previous integral can be written as

$$
\begin{aligned}
& \int_{\substack{\text { finite } \\
\text { lattice }}} \Phi_{-k^{\prime} m}^{*}(-x) \Phi_{k n}(x) d x \\
& \quad=D_{N^{\prime}}(\Delta k D) \int_{-D / 2}^{D / 2} U_{-k^{\prime} m}^{*}(-x) U_{k n}(x) e^{i \Delta k x} d x .
\end{aligned}
$$

In order to derive the orthogonality condition in an infinite lattice we have to evaluate Eq. (8) in the limit $N^{\prime} \rightarrow \infty$ : $\int_{-\infty}^{+\infty} \Phi_{-k^{\prime} m}^{*}(-x) \Phi_{k n}(x) d x=\lim _{N^{\prime} \rightarrow \infty}\left\{D_{N^{\prime}}(\Delta k D)\right\} \int_{-D / 2}^{D / 2}$
$U_{-k^{\prime} m}^{*}(-x) U_{k n}(x) e^{i \Delta k x} d x$. From the Poisson summation formula [22] one can show that the Dirichlet kernel becomes a comb of equally spaced Dirac delta functions $\delta(x)$, namely: $\lim _{N^{\prime} \rightarrow \infty}\left\{D_{N^{\prime}}(x)\right\}=2 \pi \sum_{n=-\infty}^{+\infty} \delta(x-2 \pi n)$. Therefore the inner product over the whole infinite lattice takes the form:

$$
\begin{aligned}
& \int_{-\infty}^{+\infty} \Phi_{-k^{\prime} m}^{*}(-x) \Phi_{k n}(x) d x \\
& \quad=2 \pi \sum_{q=-\infty}^{+\infty} \delta(\Delta k D-2 \pi q) \int_{-D / 2}^{D / 2} \Phi_{-k^{\prime} m}^{*}(-x) \Phi_{k n}(x) d x .
\end{aligned}
$$

Since we restrict the values of the Bloch wave number only in the first Brillouin zone (reduced zone scheme), i.e., $-\pi / D \leqslant k<\pi / D$ it follows that $-2 \pi<\Delta k D<2 \pi$. From the comb series of Eq. (9), we find that only the central term (for $q=0)$ remains. This means that $\int_{-\infty}^{+\infty} \Phi_{-k^{\prime} m}^{*}(-x) \Phi_{k n}(x) d x=$ $2 \pi \delta(\Delta k D) \int_{-D / 2}^{D / 2} \Phi_{-k^{\prime} m}^{*}(-x) \Phi_{k n}(x) d x$, and since $\delta(a k)=$ $\delta(k) /|a|$, we finally reduced the inner product calculation to one individual cell: $\int_{-\infty}^{+\infty} \Phi_{-k^{\prime} m}^{*}(-x) \Phi_{k n}(x) d x=$ $\frac{2 \pi}{D}\left[\int_{-D / 2}^{D / 2} \Phi_{-k^{\prime} m}^{*}(-x) \Phi_{k n}(x) d x\right] \delta\left(k-k^{\prime}\right)$. By combining this 
last relation with the orthonormality condition in one individual cell given by Eq. (7), we arrive at the orthonormality condition in an infinite $\mathcal{P} \mathcal{T}$ lattice:

$$
\int_{-\infty}^{+\infty} \Phi_{-k^{\prime} m}^{*}(-x) \Phi_{k n}(x) d x=\frac{2 \pi}{D} d_{k n} \delta_{n, m} \delta\left(k-k^{\prime}\right) .
$$

It is worth mentioning that this orthonormality condition is very different than the usual one used for real periodic potentials. Here both the spatial coordinate $x$ and the Bloch wave vector $k$ must be reflected around the corresponding axis of symmetry.

\section{PROJECTION COEFFICIENTS IN AN INFINITE $\mathcal{P} \mathcal{T}$ LATTICE}

It is well known that any function can always be decomposed on a complete basis. This process is straightforward if the basis happens to be orthogonal. If, on the other hand, the eigenvectors are skewed as in the case of $\mathcal{P} \mathcal{T}$ lattices, this decomposition becomes more involved. In this section we analyze this problem for infinite $\mathcal{P} \mathcal{T}$ lattice and we derive and an expression for the projection coefficients.

Let us assume an infinite optical $\mathcal{P} \mathcal{T}$ lattice with an arbitrary input field $H(x)$. This profile can be expressed as a linear superposition of the new orthonormal basis $\left\{\Phi_{k n}(x)\right\}$ associated with the array and the projection coefficients $A_{n}(k)$ of this expansion can be uniquely determined. More specifically, any beam profile $H(x, z)$ can be written as a summation of the Floquet-Bloch modes of all bands and of all Bloch wave numbers belonging to the first Brillouin zone. This can be done via the eigenmode expansion $H(x, z)=\sum_{n=1}^{+\infty} \int_{-\pi / D}^{\pi / D} A_{n}(k) \Phi_{k n}(x) \exp \left[i \beta_{n}(k) z\right] d k$. By multiplying both sides with the corresponding conjugate pair $\Phi_{-k^{\prime} m}^{*}(-x)$ and by applying the orthonormality condition of Eq. (10), we get

$$
\begin{aligned}
& \int_{-\infty}^{+\infty} \Phi_{-k^{\prime} m}^{*}(-x) H(x, z) d x \\
& =\sum_{n=1}^{+\infty} \int_{-\pi / D}^{\pi / D} A_{n}(k)\left(\int_{-\infty}^{+\infty} \Phi_{-k^{\prime} m}^{*}(-x) \Phi_{k n}(x) d x\right) \\
& \quad \times \exp \left[i \beta_{n}(k) z\right] d k \Rightarrow \\
& \int_{-\infty}^{+\infty} \Phi_{-k^{\prime} m}^{*}(-x) H(x, z) d x=\frac{2 \pi}{D} \sum_{n=1}^{+\infty} d_{k^{\prime} n} \delta_{n, m} A_{n}\left(k^{\prime}\right) \\
& \quad \times \exp \left[i \beta_{n}\left(k^{\prime}\right) z\right]
\end{aligned}
$$

and the projection coefficients are given by the formula (obviously $d_{k n}^{-1}=d_{k n}$ )

$$
A_{n}(k)=\frac{D}{2 \pi} d_{k n} \exp \left[-i \beta_{n}(k) z\right] \int_{-\infty}^{+\infty} \Phi_{-k n}^{*}(-x) H(x, z) d x .
$$

The completeness of the FB mode basis is directly related to the Parseval's identity $[21,22]$ (different from that in real lattices):

$$
\begin{gathered}
H(x, z) H^{*}(-x, z)=\left[\sum_{n=1}^{+\infty} \int_{-\pi / D}^{\pi / D} A_{n}(k) \Phi_{k n}(x) \exp \left[i \beta_{n}(k) z\right] d k\right]\left[\sum_{m=1}^{+\infty} \int_{-\pi / D}^{\pi / D} A_{m}^{*}\left(-k^{\prime}\right) \Phi_{-k^{\prime} m}^{*}(-x) \exp \left[-i \beta_{m}\left(-k^{\prime}\right) z\right] d k^{\prime}\right] \Rightarrow \\
Q=\sum_{n=1}^{+\infty} \sum_{m=1}^{+\infty} \int_{-\pi / D}^{\pi / D} \int_{-\pi / D}^{\pi / D} A_{n}(k) A_{m}^{*}\left(-k^{\prime}\right)\left(\int_{-\infty}^{+\infty} \Phi_{-k^{\prime} m}^{*}(-x) \Phi_{k n}(x) d x\right) \exp \left[i \beta_{n}(k) z-i \beta_{m}\left(k^{\prime}\right) z\right] d k d k^{\prime} \stackrel{(10)}{\Rightarrow} \\
Q=\frac{2 \pi}{D} \sum_{n=1}^{+\infty} \int_{-\pi / D}^{\pi / D} d_{k n} A_{n}(k) A_{n}^{*}(-k) d k
\end{gathered}
$$

where we define the quasipower [23] as $Q \equiv \int_{-\infty}^{+\infty} H(x, z)$ $H^{*}(-x, z) d x$ and the integrated power as $P \equiv$ $\int_{-\infty}^{+\infty} H(x, z) H^{*}(x, z) d x$. It is noteworthy to highlight that since the projection coefficients $A_{n}(k)$ are not dependent on $z$, it is easy to understand from Eq. (12) that the quasipower $Q$ is a conserved quantity in a $\mathcal{P} \mathcal{T}$ lattice, while the usual power $P$ is not. Moreover, it is straightforward to see from Eq. (11) that in general $A_{n}(k) \neq A_{n}^{*}(-k)$ and the Parseval's identity in a $\mathcal{P} \mathcal{T}$ lattice is not in any sense a trivial generalization of the one we have in a real periodic potential $P=\frac{2 \pi}{D} \sum_{n=1}^{+\infty} \int_{-\pi / D}^{\pi / D}\left|A_{n}(k)\right|^{2} d k$ [24]. In a similar manner, the closure relationship (which is formally equivalent to the Parserval's identity) in a $\mathcal{P} \mathcal{T}$ lattice can be be shown to be

$$
\delta\left(x-x^{\prime}\right)=\frac{D}{2 \pi} \sum_{n=1}^{+\infty} \int_{-\pi / D}^{\pi / D} d_{k n} \Phi_{-k n}^{*}\left(-x^{\prime}\right) \Phi_{k n}(x) d k .
$$

The above results are derived for infinite $\mathcal{P} \mathcal{T}$ optical lattices. However, from the computational point of view, one always deals with a finite domain and thus it is useful to reformulate the above analysis for finite lattices with periodic boundary conditions and derive the corresponding relations. The physics is the same in the limit of many cells (the $\int_{-\pi / D}^{\pi / D} d k$ must be now replaced by the $\sum_{k}$ ) but the computations are much easier.

\section{ORTHONORMALITY AND PROJECTION IN A FINITE $\mathcal{P} \mathcal{T}$-LATTICE}

In this paragraph we analytically examine the FB mode properties of a finite $\mathcal{P} \mathcal{T}$ symmetric periodic potential where periodic boundary conditions are imposed at the endpoints of the lattice. This approach provides a straightforward numerical implementation and calculation of the 
corresponding projection coefficients. The singularities of Dirac functions in the orthogonality conditions of Eq. (10) do not exist anymore and are replaced by a Kronecker delta. In the limit $N \rightarrow \infty$ the results approach that of the associated infinite lattice. Note that this methodology is widely used in the study of periodic crystals in solid state physics [25].

Let us assume a finite lattice of $N$ number of cells, with $L=N D$. We here apply periodic boundary conditions at the end points of the lattice $\Phi_{k n}(-L / 2)=\Phi_{k n}(L / 2)$. By using the Floquet-Bloch theorem and the periodicity of every $\mathrm{FB}$ mode we get $\exp (i k L)=1 \Rightarrow k_{j}=(2 \pi j) / L, j=$ $0, \pm 1, \pm 2, \ldots$ But these discrete values of the Bloch wave number must belong to the first Brillouin zone $k \in[-\pi / D, \pi / D)$. This leads to the following results: $k=$ $\frac{2 \pi}{L}\left[\begin{array}{lllllllll}-N^{\prime} & -N^{\prime}+1 & \cdots & -1 & 0 & 1 & \cdots & N^{\prime}-1 & N^{\prime}\end{array}\right]$, for odd number of cells $N=2 N^{\prime}+1$, and $k=$ $\frac{2 \pi}{L}\left[\begin{array}{lllllllll}-\frac{N}{2} & -\frac{N}{2}+1 & \cdots & -1 & 0 & 1 & \cdots & \frac{N}{2}-2 & \frac{N}{2}-1\end{array}\right]$ for even number of cells. It is easy to see that in the first case where $N$ is odd, the discrete $k$-spectrum is symmetric around $k=0$ and $k \in(-\pi / D, \pi / D)$, whereas in the case of $N$ being even the $k$-spectrum is asymmetric by one point at $k=-\pi / D$ and $k \in[-\pi / D, \pi / D)$. This asymmetry in the case of even number of cells leads to fundamental numerical problem, since in this case the FB mode $\Phi_{-\frac{\pi}{D}, n}(x)$ does not have a corresponding conjugate pair $\Phi_{\frac{\pi}{D}, n}^{*}(-x)$. For this reason we choose a finite lattice of odd number of cells $N=2 N^{\prime}+1$, with a symmetric $k$-spectrum.

The relation between the $\mathcal{P} \mathcal{T}$-inner product of two different FB modes in a finite lattice (with no imposed periodic boundary conditions) with that in a single cell has been already presented in Eq. (8). Here we study how the discretization of Bloch momentum $k$ (due to the imposed boundary conditions) affects this relation. In order to do that we have first to calculate the Dirichlet kernel $D_{N^{\prime}}(\Delta k D)$ of the discrete Bloch wave number $k=$ $\frac{2 \pi}{L}\left[\begin{array}{lllllllll}-N^{\prime} & -N^{\prime}+1 & \cdots & -1 & 0 & 1 & \cdots & N^{\prime}-1 & N^{\prime}\end{array}\right]$. It is known [21] that the closed form of the Dirichlet kernel is given by the following relation:

$$
\begin{aligned}
& D_{N^{\prime}}(\Delta k D) \\
& \quad= \begin{cases}\frac{\sin \left[\left(N^{\prime}+\frac{1}{2}\right) \Delta k D\right]}{\sin (\Delta k D / 2)}, & \text { when } \Delta k D \neq 0, \pm 2 \pi, \pm 4 \pi, \ldots . \\
2 N^{\prime}+1, & \text { otherwise }\end{cases}
\end{aligned}
$$

For the above discretization of the Bloch momentum $k$, it is straightforward to show that $\Delta k D=\frac{2 \pi}{N} s$, where the integer $s$ takes the values $-2 N^{\prime} \leqslant s \leqslant 2 N^{\prime}$. Since $N=2 N^{\prime}+1>2 N^{\prime}$ it follows that $\Delta k D$ becomes zero only for $s=0$ and is always true that $\Delta k D \neq 0, \pm 2 \pi, \pm 4 \pi, \ldots$ for any other non-zero value of the $s$ integer. So when $\Delta k D=0$, then $D_{N^{\prime}}(0)=$ $2 N^{\prime}+1$ from the above formula. On the other hand, when $\Delta k D \neq 0$ (with the $\sin (\Delta k D / 2)$ denominator well defined), it is easy to see that $\sin \left[\left(N^{\prime}+\frac{1}{2}\right) \Delta k D\right]=0$. By combining these two last results one can arrive at the conclusion $D_{N^{\prime}}(\Delta k D)=$ $N \delta_{k, k^{\prime}}$. By substituting this relation into Eq. (8), and applying the orthonormality condition in a single cell given by Eq. (7), we get the orthonormality condition between two FB modes of different bands with different Bloch momenta in a finite lattice with imposed periodic boundary conditions, that is

$$
\int_{\substack{\text { finite } \\ \text { lattice }}} \Phi_{-k^{\prime} m}^{*}(-x) \Phi_{k n}(x) d x=N d_{k n} \delta_{n, m} \delta_{k, k^{\prime}} .
$$

One convenient normalization for the FB modes at this point is $\bar{\Phi}_{k n}(x)=\Phi_{k n}(x) / \sqrt{N}$. As in the case of an infinite lattice, an arbitrary optical beam $f(x, z)$ can be expanded in terms of the finite number of FB modes (the number of FB modes is equal to the number of the cells of the lattice and also equal to the dimension of the discrete Bloch wave number $k$ matrix) as $f(x, z)=\sum_{n=1}^{+\infty} \sum_{m=-N^{\prime}}^{N^{\prime}} A_{n, k_{m}} \bar{\Phi}_{k n}(x) \exp \left(i \beta_{n, k_{m}} z\right)$, where $A_{n, k_{m}}$ are the projection coefficients. By applying the orthonormality condition of Eq. (14), one can directly derive formulas for the projection coefficients and for the Parseval's identity. These are given by the following equations, (15) and (16), respectively.

$$
\begin{aligned}
& A_{n, k_{m}}=d_{k_{m} n} \exp \left(-i \beta_{n k_{m}} z\right) \int_{\substack{\text { finite } \\
\text { lattice }}} \bar{\Phi}_{-k n}^{*}(-x) f(x, z) d x, \\
& \int_{\substack{\text { fintite } \\
\text { latice }}} f^{*}(-x, z) f(x, z) d x=\sum_{n=1}^{+\infty} \sum_{m=-N^{\prime}}^{N^{\prime}} d_{k_{m} n} A_{n, k_{m}} A_{n,-k_{m}}^{*} .
\end{aligned}
$$

The advantage of this approach is that all the relations involved can be numerically checked since the spectrum in the $k$-space is discrete and has finite number of FB modes. This in turn allows us to express any beam profile as a linear superposition of FB modes and thus determine the energy content of this beam in every transmission band. As we will see, this information is useful in explaining the dynamical behavior and propagation characteristics of optical beams in $\mathcal{P} \mathcal{T}$-periodic potentials.

At this point, we like to note that all the numerical results of the next two paragraphs regarding projection coefficients have been obtained by using a finite lattice.

\section{POWER OSCILLATIONS AND PHASE SINGULARITIES IN $\mathcal{P} \mathcal{T}$-OPTICAL LATTICES}

The most interesting aspects associated with $\mathcal{P} \mathcal{T}$ symmetric lattices are revealed during dynamic beam evolution. Let us consider a wide (covers several channels) Gaussian beam at an arbitrary angle of incidence. Because of the nonorthogonality of the associated FB modes and the physical nonreciprocity of the lattice, we are expecting asymmetric diffraction patterns, such as double refraction and secondary emissions [12]. For the specific periodic $\mathcal{P} \mathcal{T}$ potential $V(x)=$ $4\left[\cos ^{2}(x)+i V_{0} \sin (2 x)\right]$ that we consider in this paper, we examine the spatial evolution of a wide incident beam. In Figs. 3(a) and 3(b) we can see the intensity distributions for two different angles of incidence, when $V_{0}=0.49$. The first one in Fig. 3(a) the diffraction pattern has a crescent-like form and is symmetric around $x=0$ axis. On the other hand, in Fig. 3(b) the beam is splitting into three different components and the evolution is highly asymmetric. For a better understanding, we have calculated the beam's "energy content" for various bands. By applying Eq. (11), the stationary 

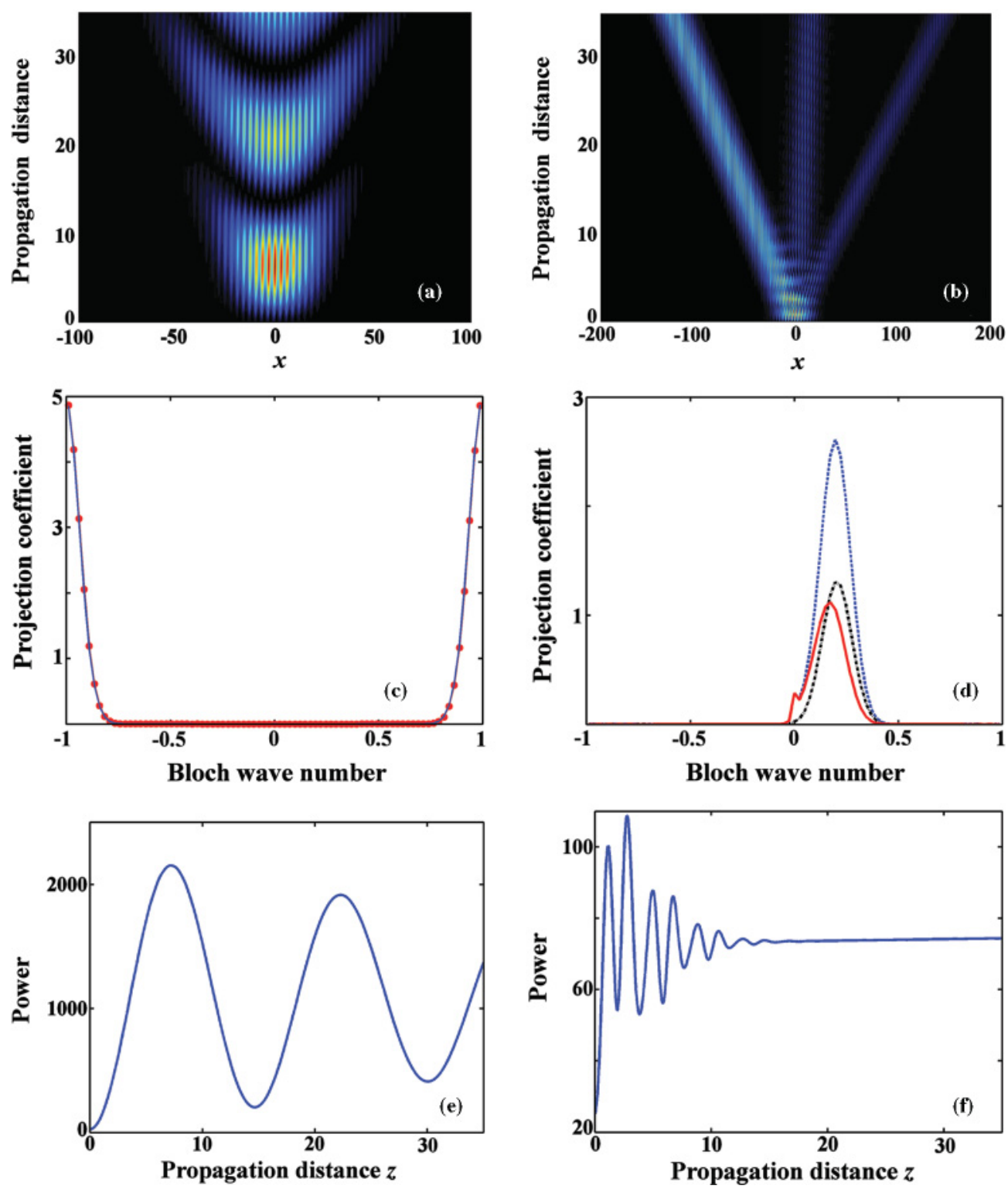

FIG. 3. (Color online) Peculiar diffraction patterns in a $\mathcal{P} \mathcal{T}$-symmetric lattice under wide beam excitation, for $V_{0}=0.49$ (below the phase transition point). Intensity evolution of two different input beams, which lead to (a) crescent-like pattern, and (b) three-beam splitting. The corresponding projection coefficient diagrams for these two input beams are depicted for the (c) first band (solid line), second band (dotted line), and (d) first band (dashed-dotted line), second band (dotted line), third band (solid line), respectively. The integrated power oscillations, with respect to the propagation distance, (e), (f) for the input beams of (a), (b), respectively.

projection coefficients $\left|A_{n}(k)\right|$ can be numerically evaluated for the first three bands (where most of the "energy" of the beam is distributed). In Figs. 3(c) and 3(d) the corresponding projection coefficient distributions for the input field of Figs. 3(a) and 3(b), respectively, are illustrated. In Fig. 3(c) the "energy" distribution for the first two bands is symmetric with respect to the center of the Brillouin zone $k=0$, and this is the reason for the symmetry of the resulted diffraction pattern of Fig. 3(a). Notice also that both bands have an equal contribution. For the input beam of Fig. 3(b), the projection coefficients for the first three bands are highly asymmetric. We can see that the most of the "energy" of the beam is distributed along the second band. The first and the third bands have similar secondary contributions. By taking this plot into account, one can easily explain the splitting of the beam in three new beams. The main left lobe is a result of the second band and the other two of the first and third band.

As we mentioned in a previous paragraph, the power $P$ is not a conserved quantity in a $\mathcal{P} \mathcal{T}$ symmetric optical lattice. So the purpose here is to understand the behavior of power with respect to the propagation distance $z$. This can be analytically done by calculating the integrated power with respect to the usual inner product $\langle h, g\rangle=\int_{-\infty}^{+\infty} h^{*}(x) g(x) d x$. The nonorthogonality of the involved FB modes plays a crucial role here. From the analysis presented in Sec. IV, it is easy to see what will be the corresponding relation for the integral $\int_{\text {cell }} \Phi_{k m}^{*}(x) \Phi_{k n}(x) d x$ in one lattice cell. The following relationship [derived in an analogous way as the orthonomality 
condition of Eq. (10)] also holds over the whole lattice:

$$
\begin{aligned}
& \int_{-\infty}^{+\infty} \Phi_{k^{\prime} m}^{*}(x) \Phi_{k n}(x) d x \\
& \quad=\frac{2 \pi}{D}\left(\int_{-D / 2}^{+D / 2} \Phi_{k m}^{*}(x) \Phi_{k n}(x) d x\right) \delta\left(k-k^{\prime}\right) .
\end{aligned}
$$

We are now in position to calculate the power of an arbitrary input beam, by expressing it as a linear superposition of nonorthogonal FB modes. The resulted formula is

$$
\begin{aligned}
P= & \frac{2 \pi}{D} \sum_{n=1}^{+\infty} \sum_{m=1}^{+\infty} \int_{-\pi / D}^{+\pi / D} A_{n}(k) A_{m}^{*}(k) \\
& \times\left(\int_{-D / 2}^{+D / 2} \Phi_{k m}^{*}(x) \Phi_{k n}(x) d x\right) \exp \left[i \Delta \beta_{n m}(k) z\right] d k
\end{aligned}
$$

where we used Eq. (14), and $\Delta \beta_{n m}(k) \equiv \beta_{n}(k)-\beta_{m}(k)$. From previous considerations we finally obtain

$$
\begin{aligned}
P= & \frac{2 \pi}{D} \sum_{n=1}^{+\infty} \int_{-\pi / D}^{\pi / D}\left|A_{n}(k)\right|^{2}\left(\int_{-D / 2}^{+D / 2}\left|\Phi_{k n}(x)\right|^{2} d x\right) d k \\
& +\frac{2 \pi}{D} \sum_{\substack{n=1 \\
n \neq m}}^{+\infty} \sum_{m=1}^{+\infty} \int_{-\pi / D}^{+\pi / D} A_{n} A_{m}^{*}\left(\int_{-D / 2}^{+D / 2} \Phi_{k m}^{*}(x) \Phi_{k n}(x) d x\right) \\
& \times \exp \left[i \Delta \beta_{n m}(k) z\right] d k .
\end{aligned}
$$

The first term represents the usual power-spectral summation that appears in real lattice. Under the usual inner product and orthogonality relations in a real lattice, the second term of Eq. (18) is zero and $\int_{-D / 2}^{+D / 2}\left|\Phi_{k n}(x)\right|^{2} d x=1$. So the above relation is reduced to the Parseval's identity in a real lattice [24]. In a $\mathcal{P} \mathcal{T}$ lattice, the FB modes are not orthogonal and hence some interference effects are expected. This interference between "skewed" FB modes is described by the second term of Eq. (18). Because of this term the power $P$ oscillates in $z$. The oscillations are not periodic in principle. The fact that the integrated power oscillates with distance is even more surprisingly given the fact that the eigenvalue spectrum is entirely real (below $\mathcal{P} \mathcal{T}$-phase transition point). In order to develop a better understanding of this behavior, let us consider two specific examples of power oscillations in a $\mathcal{P} \mathcal{T}$ - periodic potential of the form $V(x)=4\left[\cos ^{2}(x)+i V_{0} \sin (2 x)\right]$, with $V_{0}=0.49$, that is excited by a wide optical beam. More specifically, we are interested in two different cases, the first of which is when the input beam profile leads to strong power oscillations and second is exactly the opposite. In both situations, the potential parameters are kept fixed. The intensity evolution patterns of these two beams are illustrated in Figs. 3(a) and 3(b), respectively. Notice that these patterns can be spatially symmetric or asymmetric, depending on their modal "energy" distributions among the various bands. Figures 3(e) and 3(f) depict the power oscillations of the first [Fig. 3(a)] and the second beam [Fig. 3(b)].
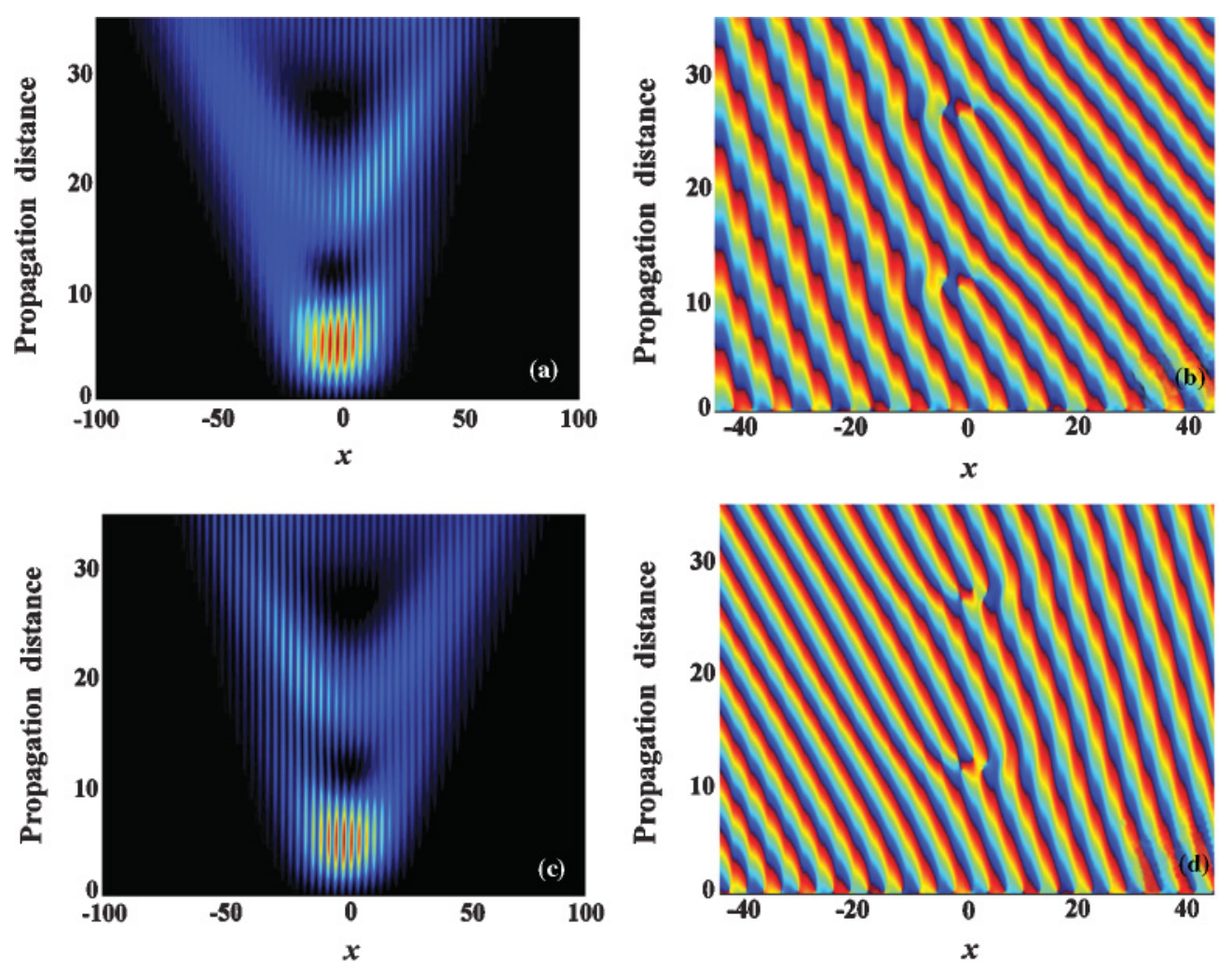

FIG. 4. (Color online) Diffraction patterns under wide beam excitation of a $\mathcal{P} \mathcal{T}$-symmetric lattice for $V_{0}=0.49$. The evolution of (a) the intensity and (b) the phase of the diffracted field, with respect to the propagation distance are shown. Notice the two phase singularities at the points where the field is zero. The phase at these points is undetermined. (c) Intensity pattern of a different input beam, and (d) phase of the corresponding propagating field (c). The phase singularities here have "topological charge" of opposite sign than these illustrated on (b). 
Another interesting feature of wave propagation in $\mathcal{P} \mathcal{T}$ symmetric periodic potentials is the existence of phase dislocations in the evolution pattern. More specifically, we examine the diffraction of a wide tilted Gaussian beam propagating in the same lattice define before. Figures 4(a) and 4(b) show the intensity and the phase of the diffracted field, respectively. When the beam splits in two different parts [Fig. 4(a)], then the field at this point is zero. Consequently, the phase is undetermined and singularities appear [26]. In the specific case, we have two phase dislocations with the same "topological charge" [Fig. 4(b)]. On the other hand, when the tilt of the beam slightly changes we get similar diffraction pattern [Fig. 4(c)], but the "topological charge" of the phase singularities has opposite sign than that in Fig. 4(b) [Fig. 4(d)].
It is noteworthy here that such characteristics and diffraction patterns have no analog whatsoever in real Hermitian optical lattices.

\section{CONCLUSIONS}

We have demonstrated that $\mathcal{P} \mathcal{T}$-symmetric optical periodic potentials can exhibit new behavior. Nonreciprocity, abrupt phase transitions and "skewness" of the associated FB modes characterize such systems. The orthonormality and projections relations, based on a new type of conjugate pairs, were analytically derived. Beam dynamics in these structures reveals that power oscillations and peculiar diffraction patterns exhibiting phase singularities are possible.
[1] C. M. Bender and S. Boettcher, Phys. Rev. Lett. 80, 5243 (1998).

[2] C. M. Bender, D. C. Brody, and H. F. Jones, Phys. Rev. Lett. 89, 270401 (2002); C. M. Bender, Am. J. Phys. 71, 1095 (2003); Z. Ahmed, Phys. Lett. A 282, 343 (2001).

[3] C. M. Bender, D. C. Brody, H. F. Jones, and B. K. Meister, Phys. Rev. Lett. 98, 040403 (2007); S. Weigert, Phys. Rev. A 68, 062111 (2003).

[4] C. M. Bender, G. V. Dunne, and P. N. Meisinger, Phys. Lett. A 252, 272 (1999); H. F. Jones, ibid. 262, 242 (1999); J. K. Boyd, J. Math. Phys. 42, 15 (2001).

[5] I. Y. Goldsheid and B. A. Khoruzhenko, Phys. Rev. Lett. 80, 2897 (1998); B. Bagchi, and C. Quesne, Phys. Lett. A 273, 285 (2000); H. Markum, R. Pullirsch, and T. Wettig, Phys. Rev. Lett. 83, 484 (1999).

[6] R. Shankar, Principles of Quantum Mechanics (Plenum Press, New York, 1994).

[7] A. E. Siegman, J. Opt. Soc. Am. A 20, 1617 (2003); Lasers (University Science Books, Mill Valley, CA, 1986).

[8] E. A. Ultanir, G. I. Stegeman, and D. N. Christodoulides, Opt. Lett. 29, 845 (2004); N. K. Efremidis and D. N. Christodoulides, Phys. Rev. E 67, 026606 (2003); N. N. Akhmediev, V. V. Afanasjev, and J. M. SotoCrespo, ibid. 53, 1190 (1996).

[9] M. V. Berry and H. J. O’Dell, J. Phys. A 31, 2093 (1998); M. V. Berry, ibid. 31, 3493 (1998).

[10] D. O. Chudesnikov and V. P. Yakovlev, Laser Physics 1, 111 (1991); M. K. Oberthaler, R. Abfalterer, S. Bernet, J. Schmiedmayer, and A. Zeilinger, Phys. Rev. Lett. 77, 4980 (1996); C. Keller, M. K. Oberthaler, R. Abfalterer, S. Bernet, J. Schmiedmayer, and A. Zeilinger, ibid. 79, 3327 (1997).
[11] R. El-Ganainy, K. G. Makris, D. N. Christodoulides, and Z. H. Musslimani, Opt. Lett. 32, 2632 (2007).

[12] K. G. Makris, R. El-Ganainy, D. N. Christodoulides, and Z. H. Musslimani, Phys. Rev. Lett. 100, 103904 (2008).

[13] Z. H. Musslimani, K. G. Makris, R. El-Ganainy, and D. N. Christodoulides, Phys. Rev. Lett. 100, 030402 (2008).

[14] Z. H. Musslimani, K. G. Makris, R. El-Ganainy, and D. N. Christodoulides, J. Phys. A 41, 244019 (2008).

[15] A. Guo, G. J. Salamo, D. Duchesne, R. Morandotti, M. VolatierRavat, V. Aimez, G. A. Siviloglou, and D. N. Christodoulides, Phys. Rev. Lett. 103, 093902 (2009).

[16] C. E. Ruter, K. G. Makris, R. El-Ganainy, D. N. Christodoulides, M. Segev, and D. Kip, Nature Phys. 6, 192 (2010).

[17] D. N. Christodoulides, F. Lederer, and Y. Silberberg, Nature (London) 424, 817 (2003).

[18] D. N. Christodoulides and R. I. Joseph, Opt. Lett. 13, 794 (1988).

[19] Z. Ahmed, Phys. Lett. A 282, 343 (2001).

[20] E. Narevicius, P. Serra, and N. Moiseyev, Europhys. Lett. 62, 789 (2003).

[21] G. Bachman, L. Narici, and E. Beckenstein, Fourier and Wavelet Analysis (Springer, New York, 2000).

[22] J. S. Walker, Fast Fourier Transforms (CRC Press, Florida, 1996).

[23] B. Bagchi, C. Quesne, and M. Znojil, Mod. Phys. Lett. A 16, 2047 (2001).

[24] F. Odeh, and J. B. Keller, J. Math. Phys. 5, 1499 (1964).

[25] C. Kittel, Introduction to Solid State Physics, 7th ed. (Wiley, New York, 1995).

[26] J. F. Nye and M. V. Berry, Proc. R. Soc. London A 336, 165 (1974). 